\title{
Efficacy of eminectomy in the treatment of prolonged mandibular dislocation
}

\author{
Leandro-Eduardo Klüppel ${ }^{1}$, Sergio Olate ${ }^{2}$, Eduardo Serena-Gomez ${ }^{1}$, Márcio De Moraes ${ }^{3}$, Roger-William \\ Fernandes-Moreira ${ }^{4}$
}

${ }^{1}$ DDS, OMFS, MSc, PhD Student of Oral and Maxillofacial Surgery, Piracicaba Dental School, State University of Campinas, Brazil

${ }^{2}$ DDS, MSc, PhD Student of Oral and Maxillofacial Surgery, Piracicaba Dental School, State University of Campinas, Brazil and Professor of Oral and Maxillofacial Surgery, Medicine School, University of La Frontera, Chile

${ }^{3}$ DDS, OMFS, MSc, PhD, Associate Professor and Coordinator of the Postgraduate Program in Oral and Maxillofacial Surgery, Piracicaba Dental School, State University of Campinas, Brazil

${ }^{4}$ DDS, OMFS, MSc, PhD, Associate Professor of Oral and Maxillofacial Surgery, Piracicaba Dental School, State University of Campinas, Brazil

Correspondence:

Faculade de Odontologia de Piracicaba

Universidade Estadual de Campinas

Av. Limeira, 901

Piracicaba-SP. CEP 13414-903, Brazil

roger@fop.unicamp.br

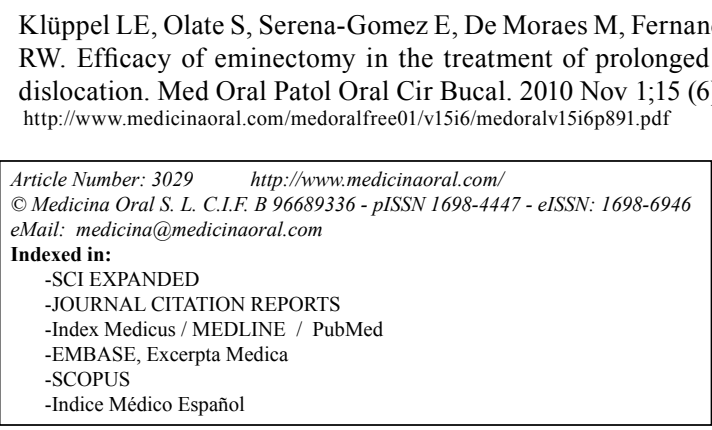

Received: $13 / 07 / 2009$

Accepted: $21 / 02 / 2010$

\section{Abstract}

Surgery of the temporomandibular joint is indicated for different clinical situations, including internal derangements, hipomobility, hipermobility, pathology and trauma. Mandibular dislocation is an acute painful condition that causes severe functional limitation. Manual reduction, with or without pharmacological assistance, is the treatment of choice and should be performed as early as possible. On rare situations mandibular dislocation may not be perceived by the patient and remain undiagnosed or misdiagnosed for a long period. This may include severe illness, neurological diseases and prolonged intensive care hospitalization with oral intubation and sedation. Treatment of prolonged mandibular dislocation is different. Morphological changes of the joint and associated structures will prevent successful manual reduction even with the patient under general anesthesia. Basically, two types of surgery may be indicated: elimination of the articular eminence (eminectomy) or reestablishment of a new condyle - ramus relationship, that can be achieved by condilotomy. This article reports a case of prolonged mandibular dislocation that was treated surgically with success. A review of the literature is performed by authors and advantages and disadvantages of each type of treatment are discussed.

Key words: Temporomandibular joint disorders, mandibular dislocations, eminectomy. 


\section{Introduction}

Internal derangements of the temporomandibular joint are represented by different clinical situations that may involve articular soft tissues, hard tissues or both. Mandibular dislocation is a condition in which condyle(s) are positioned anteriorly to the articular eminence. Despite of being uncommon, it is extremely discomfortable for the patients. When acute dislocation exists, it should be treated by manual reduction as early as possible. Not uncommonly, patients present with repeated dislocation episodes that need assistance on reduction; this is called recurrent mandibular dislocation $(1,2)$. Note that this is different from prolonged mandibular dislocation, in which the dislocated condyles are misdiagnosed or undiagnosed and left untreated.

With rare exceptions, treatment of prolonged mandibular dislocation requires surgical intervention. This is directly related to the fact that a joint that remains chronically dislocated undergoes morphological changes which is also true for periarticular tissues, especially ligaments and muscles (3-5)

Once prolonged mandibular dislocation is a rare condition, a treatment protocol is not established yet. Several treatment modalities for this rare condition have been proposed over the past decades. Advantages and disadvantages of each one will be further discussed.

The aim of this article is to report a case of chronic mandibular dislocation that was successfully treated with eminectomy. Clinical findings, surgical treatment and outcomes will be correlated with the available literature.

\section{Case Report}

A 42 year-old woman was referred for evaluation, complaining of mild facial pain, inability to occlude her teeth and difficulty in chewing and talking. Five months earlier, she was hospitalized to receive neurosurgical treatment for an aneurism of the left middle cerebral artery. At that time she was kept with intravenous sedation and mechanical ventilation (via orotraqueal intubation) for 6 days. The patients reported that when sedation was eliminated and orotraqueal intubation removed, there was a great difficult in talking and swallowing. This symptomathology, unfortunately, was misdiagnosed as a sought from the aneurism and no further investigation was performed. After 15 days of hospitalization the patient was discharged by the neurosurgical team.

Four months after being discharged, she sought dental treatment and a panoramic radiograph was ordered by her dentist who diagnosed bilateral mandibular dislocation and sent her for evaluation in the Department of Oral and Maxillofacial Surgery.

Clinical examination revealed a protruded mandible. Depressions on the region anterior to tragus were observed on both sides and condylar palpation was dif-

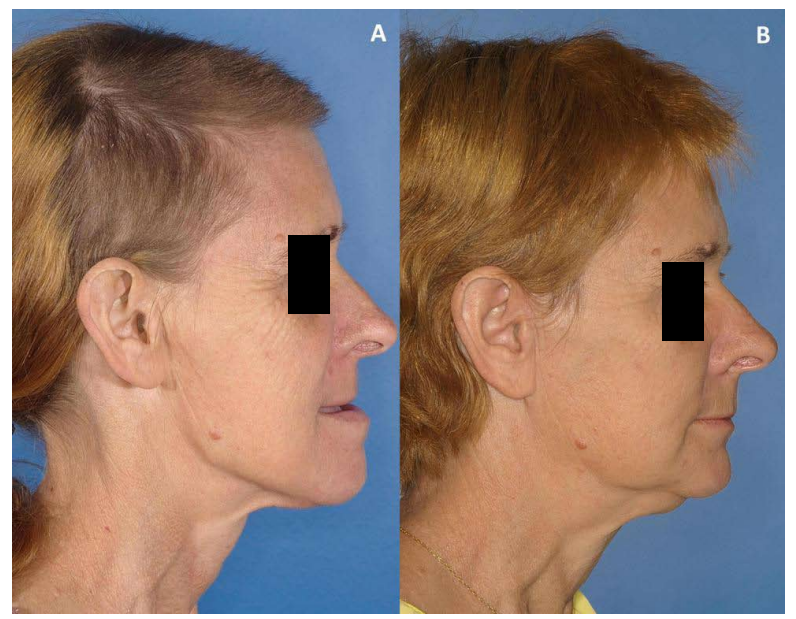

Fig. 1A. Facial appearance on initial presentation: note the false mandibular prognathism and depression on the pre-auricular area. B. 6-month postoperative facial appearance with facial harmony.

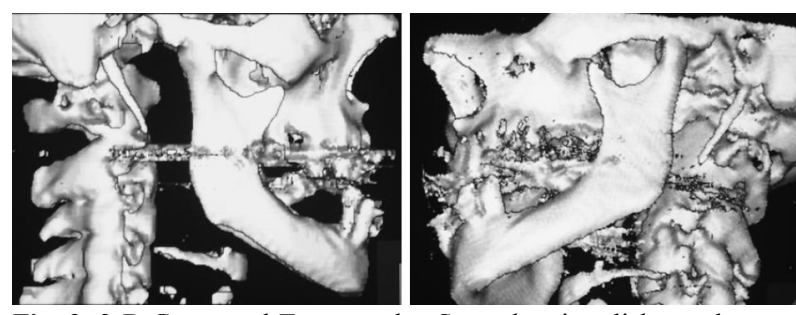

Fig. 2. 3-D Computed Tomography. Scan showing dislocated mandibular condyles.

ficult. She had no posterior teeth and a prognathic appearance was evident. The range of mandibular motion was limited in all directions and mild pain was referred in the pre-auricular area (Fig. 1).

Tomographic examination revealed that both condyles were located anteriorly to the articular eminence (Fig. 2) and a diagnosis of long-standing mandibular dislocation was made. Despite of being an exam that would offer kind information about soft tissues inside and around the joints, magnetic resonance image (MRI) was contra-indicated in this case because a metallic coil was used for embolization of the intra-cranial aneurism. There was a great risk of displacement of this metallic coil if patient was submitted to the strong magnetic field generated during MRI examination.

As a first measure, under local anesthesia and conscious sedation manual repositioning was tried without success and surgical intervention under general anesthesia was proposed.

Once general anesthesia induced muscle relaxation, a new attempt was performed and reduction could now be achieved. However, a new dislocation occurred every time mouth opening exceeded $10 \mathrm{~mm}$ (similar to what is seen on patients with recurrent mandibular dislocation). A plausible explanation for that could be that intrinsic and extrinsic ligaments of the temporomandibular joint became stretched due to the prolonged dislocation and 


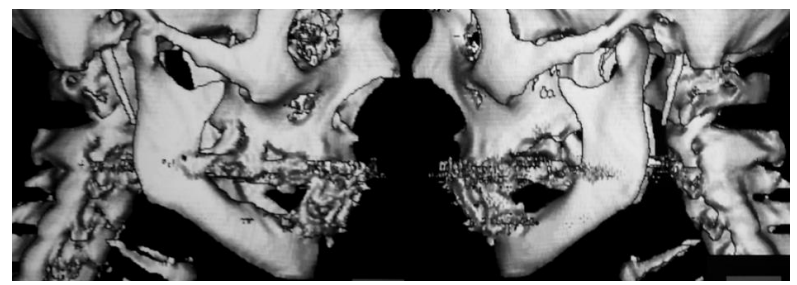

Fig. 3. Postoperative 3-D Computed Tomography Scan showing a normal relationship between the condyles and mandibular fossa allowing interincisal opening of $43 \mathrm{~mm}$ and mandibular movements without obstacles.

could not restrict anterior condylar excursion anymore. To solve this problem, eminectomy was indicated. Pre-auricular incisions were performed billateraly to promote visualization of the articular cavity, articular eminence and mandibular condyle. Eminectomy was performed with the aid of an oscilatory saw, and care was taken to remove all the irregularities, especially on the medial aspect of the eminence. On the end of this step, repositioning of the mandible could be easily performed and mouth opening could be simulated without any interference or new dislocation episode. Arch bars were applied to upper and lower teeth and elastics were used in the first postoperative week to partially restrict movements of the lower jaw. After this period and for the next 2 months, patient was instructed to perform active physiotherapy to stimulate mouth opening and excursive mandibular movements. Two weeks after surgery a solid diet was implemented without any restrictions or pain.

After 6 months interincisal opening of $43 \mathrm{~mm}$ was noted and no episode of redislocation occurred (Fig. 1). Postoperative computed tomography showed both condyles to be in normal position and the articular eminence to be flattened (Fig. 3).

\section{Discussion}

Despite of being a rare condition, prolonged mandibular dislocation requires special attention from surgeons. Treatment planning should be individualized for every patient and factors such as patient's age, general health and condition of the joint must be taken into consideration $(1,2,6)$

Conservative management of this condition (manual reduction) should be attempted initially. Unfortunately, good results are rarely obtained and surgical intervention is usually required $(4,7)$

In the presented case, dislocation probably occurred during laringoscopy for oral intubation 5 months before initial presentation, when the patient was diagnosed with an intracranial aneurism and required neurosurgery. She reported that no attempt of reduction was made while dislocation was acute. Actually, the absence of upper and lower posterior teeth masked mandibular dislocation because she did not had the classical open bite. Instead, the dislocated mandible rotated and a false prognathism developed.

Treatment of prolonged mandibular dislocation is different from acute mandibular dislocation, mainly because simple reduction is difficult to achieve or a great tendency to redislocation with minimal mouth opening occurs.

Different treatment modalities have been used for long standing mandibular dislocation. Some of them are performed in the ramus/condyle unit and other on the periarticular structures. It is the author's opinion that a mandible that remains dislocated for a long period of time induces morphological alterations in the intrinsic and extrinsic ligaments of the temporomandibular joint as well as atrophy of masticatory muscles. For this reason, after reduction is performed this patients should be treated as strong recurrent mandibular dislocation candidates.

Common procedures that involve ramus/condyle are condilectomy, condylotomy, subcondylar osteotomy with coronoidotomy, inverted L-shaped ramus osteotomy and modified vertical subsigmoid osteotomy. All of them are thought to be effective and easy to perform. However, one disadvantage is the development of malloclusion postoperatively (mainly class II with or without open bite) $(3,8,9)$. In edentulous patients this may not be a concern, but may need further correction in fully or partially dentate inviduals.

Surgery performed in the periarticular tissues is not associated with occlusal alterations. In this group, the most used procedures include eminectomy, surgical repositioning with lateral pterygoid miotomy and articular eminence augmentation with bone grafts, implants or zygomatic arch downfracturing. Another advantage of this group of procedures is related to the fact that the relationship between condyle and articular fossa is maintained $(2,4,10,11)$ Conversely, procedures performed in the ramus/condyle unit does not provide this anatomical relationship and patients become candidates to develop internal derrangement of temporomandibular joint postoperatively.

\section{References}

1. Undt G, Kermer C, Piehslinger E, Rasse M. Treatment of recurrent mandibular dislocation, Part I: Leclerc blocking procedure. Int J Oral Maxillofac Surg. 1997;26:92-7.

2. Undt G, Kermer C, Rasse M. Treatment of recurrent mandibular dislocation, Part II: Eminectomy. Int J Oral Maxillofac Surg. 1997;26:98-102.

3. Tipps SP, Landis CF. Prolonged bilateral mandibular dislocation. J Oral Maxillofac Surg. 1982;40:524-7.

4. Mizuno A, Suzuki S, Motegi K. Articular eminectomy for longstanding luxation of the mandible. Report of 2 cases. Int J Oral Maxillofac Surg. 1988;17:303-6.

5. Lee SH, Son SI, Park JH, Park IS, Nam JH. Reduction of prolonged bilateraltemporomandibular joint dislocation by midline mandibulotomy. Int J Oral Maxillofac Surg. 2006;35:1054-6. 
6. Vasconcelos BC, Porto GG, Bessa-Nogueira RV. Condylar disk plication for temporomandibular joint internal derangement treatment: surgical technique and results. Med Oral Patol Oral Cir Bucal. 2005;10 Suppl 2:E133-8.

7. Kurita K, Mukaida Y, Ogi N, Toyama M. Closed reduction of chronic bilateral temporomandibular joint dislocation. A case report. Int J Oral Maxillofac Surg. 1996;25:422-3.

8. Eppley BL, Delfino JJ. Surgical treatment of internal derangements of the temporomandibular joint: evaluation of two techniques. J Oral Maxillofac Surg. 1988;46:721-6.

9. Trumpy IG, Lyberg T. Surgical treatment of internal derangement of the temporomandibular joint: long-term evaluation of three techniques. J Oral Maxillofac Surg. 1995;53:740-6.

10. Kerstens HC, Tuinzing DB, van der Kwast WA. Eminectomy and discoplasty for correction of the displaced temporomandibular joint disc. J Oral Maxillofac Surg. 1989;47:150-4.

11. Vasconcelos BC, Porto GG, Neto JP, Vasconcelos CF. Treatment of chronic mandibular dislocations by eminectomy: follow-up of 10 cases and literature review. Med Oral Patol Oral Cir Bucal. 2009;14:e593-6. 